\title{
The Effects of Instrumental Touching on Infant Pain Perception and the Effects of Eutectic Mixture of Local Anesthetics (EMLA) on the Reduction of Pain
}

\author{
Sibel Kucukoglu ${ }^{1, *}$; Ayda Celebioglu ${ }^{1}$; ${\text { Ibrahim } \text { Caner }^{2} \text {; } \text { Gamze Ok }^{3} \text {; Rukiye Maden }}^{3}$ \\ ${ }_{1}^{1}$ Faculty of Health Science, Ataturk University, Department of Child Health and Disease Nursing, Erzurum, Turkey \\ ${ }_{3}^{2}$ Faculty of Medicine, Ataturk University, Department of Child Health and Diseases, Erzurum, Turkey \\ ${ }^{3}$ Ataturk University, Yakutiye Investigation Hospital, Newborn Clinic \\ *Corresponding author:Sibel Kucukoglu, Faculty of Health Science, Ataturk University, Department of Child Health and Disease Nursing, Erzurum, Turkey. E-mail: s_nadaroglu@hotmail.com \\ Received: April 29, 2014; Accepted: October 5, 2014
}

\begin{abstract}
Background: Premature infants, who have to spend the first week of their lives in neonatal intensive care units (NICUs), experience pain and stress in numerous cases, and they are exposed to many invasive interventions. The studies have shown that uncontrolled pain experienced during early life has negative and long-term side effects, such as distress, and such experiences negatively affect the development of the central nervous system

Objectives: The purpose of the study was to examine the effects of touching on infant pain perception and the effects of eutectic mixture of local anesthetic (EMLA) on the reduction of pain.

Patients and Methods: Data for the study were collected between March and August 2012 from the neonatal clinic of a university hospital located in eastern Turkey. The population of the study consisted of premature infants who were undergoing treatment, completed the first month and who were approved for Hepatitis B vaccine. The study consisted of two experimental groups and one control group. Information forms, intervention follow-up forms, and Premature Infant Pain Profile (PIPP) were used to collect the data. EMLA cream was applied on the vastus lateralis muscles of the first experimental group before the vaccination. The second experimental group was vaccinated by imitation (placebo), without a needle tip or medicine. Vaccination was carried out using instrumental touch in this group. A routine vaccination was applied in the control group.

Results: Mean pain scores of the group to which EMLA was applied were lower in a statistically significant way $(\mathrm{P}<0.05)$ compared to the pain scores of the other groups. Moreover, it was determined that even though invasive intervention was not applied to the newborns, the touching caused them to feel pain just as in the placebo group $(\mathrm{P}<0.005)$.

Conclusions: The results demonstrated that EMLA was an effective method for reducing pain in premature newborns, and the use of instrumental touch for invasive intervention stimulated the pain perception in the newborns.
\end{abstract}

Keywords: Pain; Nursing; Vaccination; Premature; EMLA cream

\section{Background}

Premature infants, who have to spend the first week of their lives in neonatal intensive care units (NICUs), experience pain and stress in numerous cases (1), and they are exposed to many invasive interventions (2). The studies have shown that uncontrolled pain experienced during early life has negative and long-term side effects, such as distress (3), and such experiences negatively affect the development of the central nervous system (4).

Nurses have a responsibility to use various methods to control painful practices in children in order to prevent negative long-term results of the pain and to reduce the emotional and physical effects of painful interventions (5). For effective pain management, it is important to correctly identify the pain experienced by the newborns in time. In order to reduce the pain experienced by the newborns, many pharmacological and non-pharmacological methods are used (6). With respect to the reduction of pain, the number of studies on pharmacological methods is less than the number of studies on non-pharmacological methods (7). For the pharmacological treatment of pain in newborns, opioid and non-opioid analgesic, sedatives and local anaesthetics are used (8). EMLA application, a pharmacological method that is easy to apply in the elimination and reduction of pain in newborns, is safely and effectively used for newborns undergoing intravenous (IV) vascular catheterisation, IV bloodletting, lumbar puncture, central venous catheterisation, circumcision and intramuscular (IM) injection (9, 10). In Unaldis (11) study, it was determined that EMLA cream was effective in pain

Copyright (C) 2015, Growth \& Development Research Center. This is an open-access article distributed under the terms of the Creative Commons Attribution-NonCommercial 4.0 International License (http://creativecommons.org/licenses/by-nc/4.0/) which permits copy and redistribute the material just in noncommercial usages, provided the original work is properly cited. 
reduction during painful procedures on newborns. Bozaykut et al.(12) suggested that more studies be conducted on the routine use of topical anaesthetic creams.

It is very important practicing tactile stimulation to newborns like all living. Recent studies showed that health care workers preferred instrumental touch for patients in general, expressive touch has been used very little by them. Instrumental touch is defined as having purposeful physical contact for completing a task. Expressive touch is recognized as warm, friendly physical touch and is not only for performing a duty (Morris et al. 2014 (13)). Newborns experience pain in the many of these instrumental touches therefore we think that the perception of pain can occur in newborns even without painful application during each touch.

\section{Objectives}

This study has been conducted in order to examine the effects of instrumental touching on infant pain perception and the effects of eutectic mixture of local anaesthetic (EMLA) on the reduction of pain.

\section{Patients and Methods}

\subsection{Type of Study}

The study was designed and conducted as a quasi-experimental model with both experimental and control groups.

\subsubsection{Time and Place of Study}

Data of the study were collected between March and August 2012 from the neonatal intensive care unit of a university hospital located in southern Turkey.

\subsection{Population and Sample Group of Study}

The population of the study consisted of premature infants who were undergoing inpatient treatment between specified dates, who have completed the first month, who were approved for $2^{\text {nd }}$ dose Hepatitis B vaccine and who met the study criteria.

\subsubsection{Inclusion Criteria for the Newborns}

The study received having no congenital anomaly, being around $2000 \mathrm{~g}$ and being 28 - 36 weeks old at the time of vaccination, having no analgesic or sedative intake, having no barriers to vaccination, being a stable premature and received permission from their parents.

In the study, the population also constituted the sample group without selecting a separate sample group. The size of the sample group was calculated by means of power analysis. The power of the study was 0.94 at a confidence interval of $95 \%$ and an error level of 0.05 . After determining the groups by means of drawing lots, the first 40 premature infants who met the study criteria were included in the newborn control group, the next 40 premature infants were included in the placebo group and the last 35 premature infants were included in the intervention group.

\subsection{Collection of Data}

An information form, an intervention follow-up form and the Premature Infant Pain Profile (PIPP) were used in order to collect data for the study.

\subsection{Information Form}

Prepared by researchers, the aim of this form is to collect data from the premature infants. The form includes information such as gender, gestational age, birth weight, weight and height at the end of the first month, etc.

\subsection{Intervention Follow-Up Form}

This form is prepared by researchers and contains the name and surname of the newborn, the file number and the physiological parameters before, during and after the procedure.

\subsection{PIPP}

This scale was preferred because 28 to 36 -week-old premature infants were included in the study. Developed by Stevens et al. (14), PIPP is a pain identification scale developed for 28 to 36-week-old premature newborns. Along with gestational age and behavior state, heart rate, oxygen saturation, knitting eyebrows, closing eyes, and naso-labial wrinkles are taken into consideration in this scale. For each parameter, scores are given as $0,1,2$, and 3. Validity and reliability of the scale was conducted by Derebent (6). According to PIPP, the pain of an infant is assessed over the total score. The highest score is 21 and the lowest score is 0 . If the PIPP is between 0 and 6 , the pain is at its lowest level, if it is between 7 and 12, the pain is at a medium level, and if it is between 13 and 21, the pain is at a severe level (14).

\subsection{Application of the Procedure}

The same nurse completed each vaccination, as infants may perceive pain differently if applied by different people. A nurse, by means of a monitor, recorded physiological parameters; another nurse pushed the tensionmeasuring button at the same time as the injection was applied into the skin. During the practice, a fixed camera carried out video recording, and each recording lasted for 20 - 30 seconds, on average.

Video recordings of the practices were evaluated by four specialist observers (a nurse specialising in child health and diseases, a physician specialising in newborns, a physician specialising in newborn and a pediatrician specialising in neurology). The evaluations were done independently from each other and in accordance 
with the same criteria. For each evaluation, the observers were made to watch the videos. The observers were not told which newborns were in the control group, which were in the intervention group and which were in the placebo group. They were asked to give scores on the scale according to the pain. The coefficient of concordance among observers was calculated. The concordance among the observers was found to be at a good level (kappa value $=0.65)$. Nurse who practiced in the study performed the routine vaccination by using instrumental touch in all three groups. There is no availability of pain management guideline in these clinics.

Intervention group: Each premature newborn in the intervention group received an application of $0.5 \mathrm{~g}$ EMLA cream to the vastus lateralis muscle by circular movements 30 minutes before the procedure, and the area was covered with Tegaderm. After waiting 30 minutes, alcohol of $70 \%$ was used to clean the area before vaccination, and the alcohol was let to dry. A responsible nurse, in accordance with the literature, completed the vaccination. Before, during and after the procedure, physiological parameters (heart rate, body temperature, respiration, oxygen saturation, blood pressure) were evaluated, the pain of the newborn was assessed according to the PIPP and the results were recorded on the related forms.

Placebo group: Without applying EMLA cream, using an injection or medicine and by imitating the vaccination, the procedure was applied to the children in the placebo group when they were stable and not crying. Nurse wore gloves, the cover of the incubator was opened kindly, the vastus lateralis muscle of the infant was grasped, the area was disinfected by means of alcohol $70 \%$ and the injector without a needle was touched onto the tissue and kept there for 20 seconds, on average. Before and after the procedure, physiological parameters were recorded. (Routine vaccinations practice were administered to placebo group after an hour).

Control group: With respect to the premature infants in the control group, only a routine vaccination procedure was applied not EMLA or imaginary vaccine.

\subsection{Evaluation of Data}

The data, collected in compliance with the objectives of the study, were evaluated via computer using the Statistical Package for Social Science (SPSS) 18.0 program. Percentage values, mean values, chi-square, analysis of variance, and t tests were used to assess the data.

\subsection{Ethical Principles of the Study}

During the planning process of the study, an Ethics Committee approval was received from Ataturk University, Faculty of Health Sciences, and legal permissions were obtained from the hospital where the study was conducted. Moreover, after explaining to the parents the objective of the study, the reasons for video recording and the intended purpose for such views, their approval (informed consent principle) was obtained both orally and in writing.

\section{Results}

No significant difference was determined statistically between the intervention, placebo and control groups in terms of gender, gestational age, weight and height of the infants $(P>0.05$, Table 1$)$.

$\mathrm{SaO}^{2}$ mean scores of the newborns in the intervention, placebo and control group before the vaccination were lower than the mean scores after the procedure. It was found out that the differences between the groups in heart rate mean scores after the vaccination were significant $(\mathrm{P}<0.05)$. No significant difference was determined between the experiment, placebo and control group in terms of pre and post-vaccination respiration rate, heart rate, systolic and diastolic blood pressure and temperature mean scores ( $\mathrm{P}>0.05$, Table 2$)$.

It was determined, according to mean pain scores, that while the pain of the newborns in the control group was severe, the pain of the newborns in the intervention and the placebo groups was at a medium level $(\mathrm{P}<0.05$, Table 3$)$.

Table 1. Distribution of Descriptive Characteristics of the Premature Infants

\begin{tabular}{|c|c|c|c|c|c|}
\hline Descriptive Characteristics & Experimental Group $^{a}$ & Placebo Group $^{a}$ & Control Group $^{a}$ & Test & $\mathbf{P}$ \\
\hline \multicolumn{6}{|l|}{ Gender $^{\mathrm{b}}$} \\
\hline Female & 29.7 & 25.9 & 44.4 & $\mathrm{X} 2: 5.050$ & 0.080 \\
\hline Male & 31.3 & 42.5 & 26.2 & & \\
\hline Gestational age (birth) & $30.40 \pm 2.95$ & $31.20 \pm 2.63$ & $31.20 \pm 2.63$ & $\mathrm{~F}: 1.042$ & 0.356 \\
\hline Weight (birth) & $1384.00 \pm 285.13$ & $1503.13 \pm 355.54$ & $1480.63 \pm 362.52$ & $F: 1.282$ & 0.282 \\
\hline Weight (First month) & $1918 \pm 317.35$ & $2275 \pm 1598.49$ & $2039 \pm 427.79$ & $\mathrm{~F}: 1.276$ & 0.283 \\
\hline Height, cm & $38.61 \pm 4.66$ & $40.13 \pm 2.84$ & $40.08 \pm 2.67$ & $F: 2.260$ & 0.109 \\
\hline
\end{tabular}

$\mathrm{a} X \pm \mathrm{SD}$.

b Gender values were gives as percent, SD: Standard deviation. 
Kucukoglu S et al.

It has been determined that even though no painful procedure was applied in the placebo group, $25 \%$ of new- borns felt severe pain $(\mathrm{P}<0.001$, Table 4$)$.

Flowchart of the Study has been shown in Table 5.

Table 2. Comparison of Physiological Parameters Between Three Groups Before and After the Vaccination ${ }^{\mathrm{a}}$

\begin{tabular}{|c|c|c|c|c|c|}
\hline Physiological Parameters & Experimental Intervention Group ${ }^{b}$ & Placebo Group ${ }^{b}$ & Control Group ${ }^{b}$ & Test & $\mathbf{P}$ \\
\hline \multicolumn{6}{|l|}{ Before the vaccination } \\
\hline $\mathrm{SaO}^{2}$ & $92.37 \pm 4.80$ & $92.83 \pm 4.81$ & $89.95 \pm 6.94$ & $F: 2.965$ & 0.056 \\
\hline Respiratory rate & $52.80 \pm 13.19$ & $53.30 \pm 7.90$ & $51.95 \pm 9.76$ & F: 0.173 & 0.842 \\
\hline Heart rate & $162.97 \pm 24.78$ & $165.50 \pm 18.73$ & $166.25 \pm 25.78$ & $\mathrm{~F}: 0.201$ & 0.819 \\
\hline Systolic blood pressure & $65.34 \pm 9.13$ & $70.65 \pm 9.88$ & $69.75 \pm 9.76$ & F: 3.195 & 0.051 \\
\hline Diastolic blood pressure & $41.49 \pm 6.71$ & $41.93 \pm 7.94$ & $41.48 \pm 8.46$ & F: 0.043 & 0.958 \\
\hline Temperature & $36.60 \pm 0.59$ & $36.70 \pm 0.45$ & $36.62 \pm 0.53$ & F: 0.400 & 0.671 \\
\hline \multicolumn{6}{|l|}{ After the vaccination } \\
\hline $\mathrm{SaO}^{2}$ & $92.17 \pm 6.45$ & $91.20 \pm 6.70$ & $94.50 \pm 11.54$ & F: 0.649 & 0.525 \\
\hline Respiratory rate & $55.06 \pm 17.73$ & $54.75 \pm 13.02$ & $50.15 \pm 11.24$ & F:1.483 & p:0.231 \\
\hline Heart rate & $154.74 \pm 18.33$ & $150.78 \pm 21.02$ & $166.05 \pm 18.50$ & F:6.658 & 0.002 \\
\hline Systolic blood pressure & $65.23 \pm 9.01$ & $70.03 \pm 10.17$ & $69.22 \pm 9.57$ & F: 2.613 & 0.078 \\
\hline Diastolic blood pressure & $41.94 \pm 6.67$ & $42.78 \pm 9.57$ & $41.10 \pm 8.28$ & F: 0.405 & 0.668 \\
\hline Temperature & $36.45 \pm 0.85$ & $37.18 \pm 2.09$ & $36.62 \pm 0.48$ & F: 3.073 & 0.055 \\
\hline
\end{tabular}

${ }^{\mathrm{a}} \mathrm{SaO}^{2}$ : Oxygen saturation.

b $\mathrm{X} \pm \mathrm{SD}$.

Table 3. Comparison of Mean Pain Scores of the Intervention, Placebo and Control Group

\begin{tabular}{|c|c|c|c|c|c|}
\hline Mean Pain Scores & Experimental Group $(35)^{\mathrm{a}}$ & Placebo Group $(40)^{a}$ & Control Group $(40)^{a}$ & Test (F) & $\mathbf{P}$ \\
\hline & $9.60 \pm 5.12$ & $6.13 \pm 5.97$ & $19.78 \pm 3.50$ & 81.161 & 0.000 \\
\hline
\end{tabular}

${ }^{\mathrm{a}} \mathrm{X} \pm \mathrm{SD}$.

Table 4. Comparison of Pain Levels of the Intervention, Placebo and Control Groups

\begin{tabular}{|c|c|c|c|c|c|}
\hline Pain Groups According to PIPP & Experimental Group $(35)^{\mathrm{a}}$ & Placebo Group $(40)^{a}$ & Control Group $(40)^{\mathrm{a}}$ & Test & $\mathbf{P}$ \\
\hline Mild (0 - 6 score) & $5(14.3)$ & $16(40.0)$ & $0(0.0)$ & $\mathrm{X} 2=44.780$ & 0.000 \\
\hline Moderately (7 - 12 score) & $12(34.3)$ & $14(35.0)$ & $2(5.0)$ & & \\
\hline Severe (13 - 21 score) & $18(51.4)$ & $10(25.0)$ & $38(95.0)$ & & \\
\hline
\end{tabular}

a Values are presented as No. (\%).

Table 5. Flowchart of the Study

\begin{tabular}{lcc}
\hline Intervention group & Placebo group & Control group \\
\hline $\begin{array}{l}\text { o.5 g EMLA cream applied 30 minutes } \\
\text { before the vaccination }\end{array}$ & $\begin{array}{c}\text { Imitating vaccination was applied } \\
\text { without EMLA, injection or medicine but } \\
\text { using instrumental touching }\end{array}$ & $\begin{array}{c}\text { Routine vaccinations practice was } \\
\text { administered using instrumental } \\
\text { touching. }\end{array}$ \\
$\begin{array}{l}\text { Routine vaccinations practice was } \\
\text { administered using instrumental } \\
\text { touching }\end{array}$ & $\begin{array}{c}\text { Before, during and after the procedure } \\
\text { physiological and PIPP were evaluated }\end{array}$ & $\begin{array}{c}\text { Before, during and after the procedure, } \\
\text { physiological and PIPP were evaluated }\end{array}$ \\
$\begin{array}{l}\text { Before, during and after the } \\
\text { procedurephysiological and PIPP were } \\
\text { evaluated }\end{array}$ & & \\
\hline
\end{tabular}




\section{Discussion}

The pain experienced by newborns frequently appears during invasive interventions. It has been recently documented that only $1 \%$ of infants receive pharmacologic interventions to treat pain during immunisation in clinical practices (15). Pharmacotherapy is effective in reducing the pain from medical procedures, and promising interventions for immunisation pain include topical local anesthetics $(16,17)$.

Physiological symptoms caused by painful stimulators indicate the general stress state of the body. While the most frequently used physiological symptoms, such as heart rate, blood pressure, respiration and oxygen saturation, are used in the evaluation of pain occurring due to acute procedures, hormonal and metabolic variables are used in the assessment of prolonged and chronic pain (2). Although no statistical difference was observed between the groups in terms of pre-procedural respiration, heart rate, systole, diastole and fever mean scores, a significant difference was found between groups in terms of post-procedure $\mathrm{PO}^{2}$ and body temperature, which is contrary to the literature (Table 2). In the study conducted by Ok (18), the efficacy of EMLA cream in the reduction of pain occurring during intravenous cannulation for premature infants was examined and the highest increase in peak heart rate was seen in newborns in the control group during the procedure. However, the difference between groups was not statistically significant. In other studies, it was stated that respiratory rate, heart rate, blood pressure and oxygen saturation were not significant in pain analysis before and after the procedure $(10,19)$.

Routine immunisation is the most common reason for iatrogenic pain in childhood (20). In the study, it was determined that, according to mean pain scores, the pain of newborns in the control group was severe and the pain of newborns in the intervention and placebo groups was at a medium level (Table 3). Topical local anaesthetics have been consistently shown to reduce injection pain (by 40\%) during the administration of various vaccines, including diphtheria-pertussis-tetanus (21), diphtheria-pertussis-tetanus and polio (22), diphtheriatetanus-acellular pertussis-inactivated poliovirus-Haemophilus influenzae type b conjugate, hepatitis B27 and measles-mumps-rubella (23). In the study conducted by Dilil et al. (17) infants in the lidocaine-prilocaine group cried for a shorter period compared to infants in the control group during vaccination. Taddio et al. (24) reported a significant increase in the latency of the first cry and a decrease in the duration of the first cry for infants who received lidocaine-prilocaine versus the placebo. In the study conducted by Nahum et al. (25), the mean PIPP scores were significantly lower in the EMLA group compared with the placebo group during suprapubic aspiration in young infants. Similarly, parents reported lower mean pain and crying scores in the lidocaine-pri- locaine group in comparison with the placebo group. Instrumental touch was used in this group as all groups of study. However, it was thought not to perceive this shape of the touch by infants because of the effect of the local anesthetic drug.

In the study, it was determined that even though no painful procedure was applied in the placebo group, $25 \%$ of the infants felt severe pain (Table 4 ). Preterm and term newborns, who have to spend the first week of their lives in NICUs, experience pain and stress due to various reasons. They are exposed to many procedural touches like invasive procedures without analgesia. Much of this touch in the NICU is a procedural touch, and infants born very preterm often miss out on comforting touch stimulation (Smith 2012 (26)). Morris et al. (2014) (13) also showed that a large majority of touches were instrumental and pertained to functional mobility. Among these interventions are bloodletting from the heel, venous intervention, arterial catheterisation, lumbar puncture, newborn examination, medical dressing change, gavage tube insertion, IM injection, postural drainage, removing sutures, circumcision, etc. All these painful interventions are also stressful and affect the clinical course of infants. The pain experienced by the infant may affect his/her behaviors, family-infant interaction, and adaptation with the outside world. It may also cause changes in the development of the senses and the brain as well as negatively affect growth. Newborns experience physiological and metabolic problems as well as the above-mentioned behaviors. Among them, the following can be included: excess protein consumption, electrolyte imbalance, sepsis resulting from a weak immune system, metabolic acidosis, pulmonary and cardiac failure and death (27). Untreated procedural pain in childhood was shown to have significant adverse sequelae, including the development of hypersensitivity to future pain, preprocedural anxiety because of conditioning and needle phobia (21). From this perspective, it has been shown that health care professionals should use more expressive touch when handling babies.

In supporting the development of infants, reductions in stress symptoms, increases in stability symptoms, regulations in individual care and pain control, care should be provided in NICUs. It is required to evaluate carefully the pain of newborns and to learn treatment interventions. EMLA cream is a useful adjunct to local anesthetic for newborn vaccinations because it effectively reduces the sharp pain induced by needle punctures. In addition, it has been emerged that health care professionals need to do more expressive touch.

\subsection{Limitation}

Conducting our study in only one city a neonatal intensive care unit is a limitation of this study. Another important limitation is that the study was completed with a low number of preterms. 


\section{Acknowledgements}

The authors would like to express their special thanks to the participants and the Ataturk University Hospital for their collaboration and contribution.

\section{Authors' Contributions}

All authors have carried out (design, acquisition of data, data analysis, manuscript preparation, critical revision of the manuscript) the study. All authors read and approved the final manuscript.

\section{References}

1. Hui-Chen F, Hsiu-Lin C, Shun-Line C, Tai-Ling T, Li-Jung W, Hsing IT, et al. The effect of EMLA cream on minimizing pain during venipuncture in premature infants. J Trop Pediatr. 2013;59(1):72-3.

2. Derebent E, Yigit, R. . Pain in newborn: assesment and management C.U. Hemsirelik Yüksek Okulu Derg. 2006;10(2):41-7.

3. Young KD. Pediatric procedural pain. Ann Emerg Med. 2005;45(2):160-71.

4. Gradin M, Eriksson M, Holmqvist G, Holstein A, Schollin J. Pain reduction at venipuncture in newborns: oral glucose compared with local anesthetic cream. Pediatrics. 2002;110(6):1053-7.

5. Rogers TL, Ostrow CL. The use of EMLA cream to decrease venipuncture pain in children.J Pediatr Nurs. 2004;19(1):33-9.

6. Derebent E, Yigit R. Non-pharmacological pain management in newborn. Firat University of Health Sciences Journal of Medicine. 2008;22(2):113-8.

7. Cavusoglu, H. . [Child Health Nursing]. 8 edAnkara: System Offset; 2008.

8. Weise KL, Nahata MC. EMLA for painful procedures in infants. $J$ Pediatr Health Care. 2005;19(1):42-7.

9. Stevens B, McGrath P, Gibbins S, Beyene J, Breau L, Camfield C, et al. Determining behavioural and physiological responses to pain in infants at risk for neurological impairment. Pain. 2007;127(1-2):94-102.

10. Mucignat V, Ducrocq S, Lebas F, Mochel F, Baudon JJ, Gold F. [Analgesic effects of Emla cream and saccharose solution for subcutaneous injections in preterm newborns: a prospective study of 265 injections]. Arch Pediatr. 2004;11(8):921-5.

11. Unaldi, N. The effect of otektik mix and sucrose on pain of neonates in painfull treatment Health Sciences Institute, Child Health and Nursing Department. Istanbul: Marmara University; 2009. Master Thesis.

12. Bozaykut A, Guven G, Erkum T. Methemoglobinemia secondary to topical anesthetic cream: a case report. Turkish Archives of Pediatrics. 2004;39:88-90.
13. Morris D, Henegar J, Khanin S, Oberle G, Thacker S. Analysis of touch used by occupational therapy practitioners in skilled nursing facilities. Occup Ther Int. 2014;21(3):133-42.

14. Stevens B, Johnston C, Petryshen P, Taddio A. Premature Infant Pain Profile: development and initial validation. Clin J Pain. 1996;12(1):13-22.

15. Taddio A, Manley J, Potash L, Ipp M, Sgro M, Shah V. Routine im munization practices: use of topical anesthetics and oral analgesics. Pediatrics. 2007;120(3):e637-43.

16. Cohen L, Bernard R, McClellan C, Piazza-Waggoner C, Taylor B MacLaren J. Topical Anesthesia Versus Distraction for Infants' Immunization Distress: Evaluation With 6-Month Follow-Up. Children's Health Care. 2006;35(2):103-21.

17. Dilli D, Kucuk IG, Dallar Y. Interventions to reduce pain during vaccination in infancy. J Pediatr. 2009;154(3):385-90.

18. Ok G. Evaluation of the effect of emla cream in reducing the pain of intravenous connulation in premature newborns. Erzurum: Ataturk University; 2012. Master Thesis.

19. Grabska J, Walden P, Lerer T, Kelly C, Hussain N, Donovan T, et al. Can oral sucrose reduce the pain and distress associated with screening for retinopathy of prematurity? J Perinatol. 2005;25(1):33-5.

20. Schechter NL, Zempsky WT, Cohen LL, McGrath PJ, McMurtry $\mathrm{CM}$, Bright NS. Pain reduction during pediatric immunizations: evidence-based review and recommendations. Pediatrics. 2007;119(5):e1184-98.

21. Taddio A, Nulman I, Goldbach M, Ipp M, Koren G. Use of lidocaine-prilocaine cream for vaccination pain in infants. J Pediatr 1994;124(4):643-8.

22. Cassidy KL, Reid GJ, McGrath PJ, Smith DJ, Brown TL, Finley GA A randomized double-blind, placebo-controlled trial of the EMLA patch for the reduction of pain associated with intramuscular injection in four to six-year-old children. Acta Paediatr. 2001;90(11):1329-36.

23. O'Brien L, Taddio A, Ipp M, Goldbach M, Koren G. Topical $4 \%$ amethocaine gel reduces the pain of subcutaneous measlesmumps-rubella vaccination. Pediatrics. 2004;114(6):e720-4.

24. Taddio A, Ilersich AL, Ipp M, Kikuta A, Shah V, H. ELPinKIDS Team . Physical interventions and injection techniques for reducing injection pain during routine childhood immunizations: systematic review of randomized controlled trials and quasi-randomized controlled trials. Clin Ther. 2009;31 Suppl 2:S48-76.

25. Nahum Y, Tenenbaum A, Isaiah W, Levy-Khademi F. Effect of eutectic mixture of local anesthetics (EMLA) for pain relief during suprapubic aspiration in young infants: a randomized, controlled trial. Clin J Pain. 2007;23(9):756-9.

26. Smith JR. Comforting touch in the very preterm hospitalized in fant: an integrative review. Adv Neonatal Care. 2012;12(6):349-65.

27. Reyes S. Nursing assessment of infant pain. J Perinat Neonatal Nurs. 2003;17(4):291-303. 